\title{
ARTICLE
}

\section{Azide and trans-Cyclooctene dUTPs: Incorporation into DNA Probes and Fluorescent Click-Labelling}

\author{
Xiaomei Ren, ${ }^{a}$ Afaf H. El-Sagheer ${ }^{a, b}$ and Tom Brown ${ }^{a^{*}}$
}

Cite this: DOI: $10.1039 / \times 0 \times x 00000 x$

Received 00th January 2012

Accepted 00th January 2012

DOI: $10.1039 / \times 0 \times x 00000 x$

www.rsc.org/
5-Azidomethyl dUTP and two 5-trans-cyclooctene dUTPs with different linkers between the TCO and the uracil base have been incorporated into DNA by primer extension, reversetranscription and PCR amplification. For azidomethyl dUTP the PCR reaction was successful even when the modified dUTP was not supplemented with dTTP. In one case 335 azidomethyl dU residues were incorporated into the 523 base pair amplicon using this methodology. 5-Azidomethyl dUTP was found to be a better substrate for DNA polymerases than the trans-cyclooctene dUTPs. However, the inverse electron demand Diels-Alder reaction between cyclooctene DNA and a tetrazine Cy3-dye was more efficient than the strain-promoted reaction between azide DNA and a bicyclo [6.1.0] non-4-yne Cy3 dye.

\section{Introduction}

Nascent DNA synthesis can be monitored in proliferating cells by fluorescence. Cells are incubated with the thymidine analogue 5-ethynyl-2'-deoxyuridine which is converted to its triphosphate by cellular enzymes and incorporated into genomic DNA by endogenous polymerases. Subsequent cellfixation and derivatisation of the alkyne-modified DNA with a fluorescent azide (e.g. TAMRA azide) via the CuAAC reaction $^{1,2}$ produces labelled DNA (Figure 1.A) which can be visualised by fluorescent cell imaging. ${ }^{3}$ The same approach has been adapted to detect RNA synthesis in cells by incorporating the ribonucleoside 5-ethynyluridine into newly transcribed RNA. ${ }^{4}$ The methodology is compatible with fixed cells, but the toxicity of copper precludes its use on live cells. Recently it has been shown that the need for toxic metal ion catalysis is negated if an azide-modified nucleoside is incorporated into genomic DNA and a cyclooctyne derivative of the dye ${ }^{5}$ (such as bicyclo [6.1.0] non-4-yne $(\mathrm{BCN}))^{6}$, is used in the fluorescent labelling step (Figure 1.B). ${ }^{7}$ Relief of ring strain by a change of hybridisation from $\mathrm{sp}$ to $\mathrm{sp}^{2}$ drives a fast chemical reaction between the cyclooctyne attached to the dye and the azidemodified DNA. This reaction, known as the strain-promoted alkyne-azide cycloaddition (SPAAC) reaction, ${ }^{7,8}$ has also been used to label synthetic oligonucleotides. ${ }^{9}$ Unlike bulky dyelabelled nucleosides, ethynyl $\mathrm{dU}$ and azidomethyl $\mathrm{dU}$ are sufficiently similar to their natural congener (thymidine) to be converted to their triphosphates by endogenous enzymes within cells. This intracellular conversion of nucleoside to dNTP is essential; unlike free nucleosides, dNTPs cannot be used in cell-labelling experiments as they do not pass through the cell membrane.

In addition to cell imaging, there are many applications that require labelled DNA, for which click chemistry is particularly useful. ${ }^{10}$ A popular approach is to incorporate functional groups such as alkynes into the DNA during PCR amplification, primer extension or nick translation via a modified dNTP, and to subsequently label the modified DNA with an azide derivative of a reporter group such as a fluorescent dye. ${ }^{11-14}$ Similarly, azides can be incorporated into DNA or RNA for fluorophore attachment via CuAAC, SPAAC or Staudinger reactions. ${ }^{15-18}$ Such two-step labelling processes are necessary because direct enzyme-mediated incorporation of fluorophore-labelled dNTPs during PCR is inefficient due to the bulky nature of fluorescent dyes. In contrast, dNTPs that are modified by addition of sterically undemanding groups such as terminal alkynes are incorporated much more efficiently.

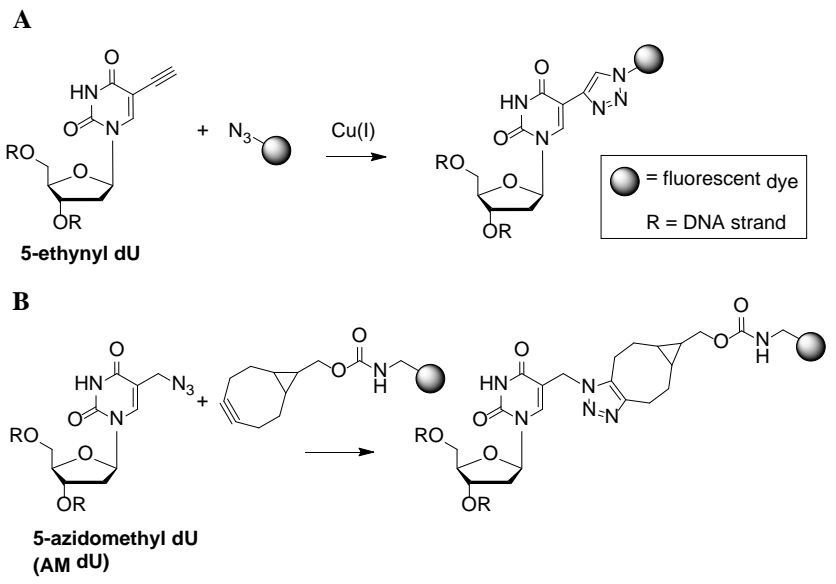

Figure 1. Fluorescent DNA labelling in cells: A). 5-Ethynyl dU and a fluorescent azide (CuAAC); B). 5-Azidomethyl dU and a BCNlabelled fluorophore (SPAAC).

Crystal structures of the thermostable DNA polymerase KlenTaq with alkyne-modified substrate dNTPs in its active site illustrate the plasticity of the enzyme and the presence of flexible regions of the dNTP binding pocket. Due to these 
factors alkyne modifications at the C5 atom of dU cause only minor disruption to the ternary complex of the enzyme. ${ }^{13,19}$ We are interested in synthesising fluorescent DNA for a variety of biological applications including those described above, and are investigating the incorporation of modified dNTPs into DNA by primer extension, reverse transcription and PCR, and determining the efficiency of subsequent labelling using various click reactions. Here we present studies on azidomethyl dUTP (AM dUTP) and two trans-cyclooctene (TCO) modified dUTP analogues with different linkers between the TCO moiety and the nucleobase.

Table 1. Oligonucleotide sequences used in primer extension, reverse transcription and PCR reactions

\begin{tabular}{ll}
\hline Code & Sequences(5'-3') \\
\hline T1 & CAGTCACTGTACTGCCGACACACATAACC \\
& (DNA template) \\
T2 & CAGTCACAAAACTGCCGACACACATAACC \\
& (DNA template) \\
T3 & CTGTCACTGTGCTGCCGACACACATAACC \\
& (DNA template) \\
P4 & FAM-GGTTATGTGTGTCGGCAG (primer ) \\
T5 & CAGUCACUGUACUGCCGACACACAUAACC \\
& (RNA template) \\
T6 & CAGUCACAAAACUGCCGACACACAUAACC \\
& (RNA template) \\
P7 & GCATTCGAGCAACGTAAG (PCR primer) \\
P8 & GGTTATGTGTGTCGGCAG (PCR primer) \\
T9 & FAM- \\
& GGTTATGTGTGTCGGCAGTATTGTCAGTGT \\
& GAATTCCAGAGTGTGAGATTGTGTCTGGC \\
& GATCTTACGTTGCTCGAATGC (PCR template) \\
P10 & GTTTGGCTTTAGAGGCTGGAG (Plasmid primer) \\
P11 & ACTGCAATACGAATAATGGCTAC (Plasmid \\
& primer) \\
T12 & Template from plasmid HydGdCTD5 (sequence in \\
& Supporting Information)
\end{tabular}

FAM is 6-carboxamidohexylfluorescein. The RNA template has the equivalent sequence to the DNA template. The bold A (A and T for PCR template) show the sites of incorporation of the modified triphosphates. All oligonucleotides were characterized by mass spectrometry.

\section{Experimental}

\section{Primer extension experiments}

In a $20 \mu \mathrm{L}$ reaction, 66 pmol of FAM labelled DNA primer (P4), 132 pmol of template (T1, T2 or T3 in Table 1) and 3.2 nmol of each modified dUTP or unmodified dNTP were mixed with the polymerase enzyme ( 1 unit for GoTaq and Klenow, 0.5 unit for KOD) and $1 \mathrm{X}$ buffer (supplied with the enzymes). In the case of KOD polymerase, $1 \mathrm{mM} \mathrm{MgCl}_{2}$ was added separately to the reaction mixture. The reaction mixtures were heated for $1.5 \mathrm{~h}$ (KOD and GoTaq at $60{ }^{\circ} \mathrm{C}$, Klenow at $37^{\circ} \mathrm{C}$ ) and $20 \mu \mathrm{L}$ formamide was added before analysis by $20 \%$ denaturing polyacrylamide gel electrophoresis (PAGE) at a constant $20 \mathrm{~W}$ of power in $1 \mathrm{X}$ TBE buffer. For the mass spectrometry analysis, two $20 \mu \mathrm{L}$ reactions were carried out, followed by ethanol precipitation. DNA was precipitated by mixing with $4 \mu \mathrm{l}$ of $3 \mathrm{M}$ sodium acetate ( $\mathrm{pH}$ 5.3) followed by $120 \mu \mathrm{l}$ ethanol. The mixture was then left on dry ice for $10 \mathrm{~min}$ and at $-20{ }^{\circ} \mathrm{C}$ for further $20 \mathrm{~min}$. This was followed by centrifugation at $4{ }^{\circ} \mathrm{C}$ for $30 \mathrm{~min}(13,000 \mathrm{rpm})$. The precipitate was dissolved in $15 \mu \mathrm{L}$ of water and analyzed on a Bruker microTOF mass spectrometer (ES mode). KOD DNA polymerase was purchased from Merck Millipore, Klenow large fragment from New England Biolabs and GoTaq from Promega.

\section{Reverse transcription}

Samples containing 66 pmol of FAM labelled DNA primer (P4), 132 pmol of RNA template (T5 and T6 in Table 1) and $3.2 \mathrm{nmol}$ modified dUTP or unmodified dNTP were mixed with reverse transcriptase (M-MuLV RT (RNase $\mathrm{H}^{-}$) 100 units, or AMV RT 5 units) and $1 \mathrm{X}$ buffer. $1 \mathrm{X}$ dithiothreitol (DTT, supplied with the enzyme) was added to the M-MuLV reaction, while 20 units of RNase inhibitor were added to AMV reactions. The final reaction volume was $20 \mu \mathrm{L}$. The reaction mixtures were heated at $42{ }^{\circ} \mathrm{C}$ for $16 \mathrm{~h} .20 \mu \mathrm{L}$ of formamide was added and samples were analyzed by $20 \%$ denaturing PAGE at a constant $20 \mathrm{~W}$ of power. For the mass spectrometry analysis, two $20 \mu \mathrm{L}$ reactions were carried out, followed by ethanol precipitation. The DNA precipitate was dissolved in 15 $\mu \mathrm{L}$ of water and analyzed by mass spectrometry. M-MuLV RT (RNase $\mathrm{H}^{-}$), AMV RT and RNase inhibitor were purchased from New England Biolabs.

\section{Polymerase chain reaction}

For template T9, samples containing $10 \mathrm{pmol}$ of two primers (P7, P8, Table 1), $50 \mathrm{pg}$ of template (T9, Table 1) and $5 \mathrm{nmol}$ modified dUTP or unmodified dNTP in total were added followed by GoTaq DNA polymerase (1 unit), 5X GoTaq buffer and SYBR Green $(0.6 \mu \mathrm{L}, 3.75 \mathrm{X})$. The final reaction volume was $20 \mu \mathrm{L}$ with $1 \mathrm{X}$ reaction buffer. For plasmid template T12, Samples containing $10 \mathrm{pmol}$ of two primers (P10, P11, Table 1), 300 pg of plasmid template (HydGdCTD5, sequence in SI) and $10 \mathrm{nmol}$ modified dUTP or unmodified dNTP in total were added followed by KOD DNA polymerase (0.5 unit), 10X KOD polymerase buffer ( $\mathrm{pH}$ 8.0), $25 \mathrm{mM}$ $\mathrm{MgCl}_{2}$ and SYBR Green $(0.6 \mu \mathrm{L}, 3.75 \mathrm{X})$. The final reaction volume was $20 \mu \mathrm{L}$ with $1 \mathrm{X}$ reaction buffer and $2 \mathrm{mM} \mathrm{MgCl}_{2}$. Amplification was performed on a real-time PCR instrument (Bio-Rad CFX96) using the following procedure: an initial denaturing at $95{ }^{\circ} \mathrm{C}$ for $2 \mathrm{~min}$, followed by 25 cycles of denaturing at $95^{\circ} \mathrm{C}$ for $30 \mathrm{~s}$, primer annealing at $54{ }^{\circ} \mathrm{C}$ for $30 \mathrm{~s}$, and extension at $72{ }^{\circ} \mathrm{C}$ for $30 \mathrm{~s}$. Further extension was carried out at $72{ }^{\circ} \mathrm{C}$ for $5 \mathrm{~min}$. This was followed by melting experiments involving heating the amplification mixture to 95 ${ }^{\circ} \mathrm{C}$ for $30 \mathrm{~s}$, cooling to $30^{\circ} \mathrm{C}$, then increasing the temperature at 1 or $0.5^{\circ} \mathrm{C}$ per second to $95^{\circ} \mathrm{C}$ and holding at each temperature for $5 \mathrm{~s}$. The samples were analyzed by $1.5 \%$ or $2 \%$ agarose gel electrophoresis with $20 \mu \mathrm{L}$ of ethidium bromide $(1 \mathrm{mg} / \mathrm{ml})$. Gel was run at constant voltage $(126 \mathrm{~V})$ in $1 \mathrm{X}$ TBE buffer.

\section{Fluorescent labelling}

Two $20 \mu \mathrm{L}$ primer extension reactions were performed as explained in the previous section using AM, TCO dUTPs, or unmodified dNTP negative control. Ethanol precipitation of the 
product was carried out after primer extension reaction. The precipitate was re-dissolved in $40 \mu \mathrm{L}$ of $1 \mathrm{X}$ GoTaq Green buffer and $20 \mu \mathrm{L}$ of the AM dUTP extension product was reacted with 6.4 nmol Cy3-bicyclo [6.1.0] non-4-yne (Cy3BCN). Similarly $20 \mu \mathrm{L}$ of TCO dUTP products were reacted with 6.4 nmol 6-methyl-tetrazine-sulfo-Cy3. When the reaction was complete, another ethanol precipitation was performed. 20 $\mu \mathrm{l}$ of formamide was added to the labelled and unlabelled primer extension reactions followed by analysis on a $20 \%$ PAGE gel at a constant $20 \mathrm{~W}$ of power. For the mass spectrometry analysis, two $20 \mu \mathrm{L}$ labelling reactions were carried out, followed by gel-filtration on a NAP-10 column.

For PCR reactions, products were first extracted from agarose gel using a Qiagen gel extraction kit according to the manufacturer's instructions. The solution was quantified using a Nanodrop 1000 UV spectrometer. $460 \mathrm{ng}$ of T9 products or $736 \mathrm{ng}$ of T12 products were diluted to $40 \mu \mathrm{L} 1 \mathrm{X}$ GoTaq Green buffer solution. Next, $20 \mu \mathrm{L}$ of each solution was labelled with $10 \mathrm{nmol}$ of 6-methyl-tetrazine-sulfo-Cy3 for $30 \mathrm{~min}$. After the labelling reactions, ethanol precipitation was applied to remove extra free dyes. Samples were analyzed by $1.5 \%$ or $2 \%$ agarose gel electrophoresis at constant voltage $(126 \mathrm{~V})$ in $1 \mathrm{X}$ TBE buffer. The gel was photographed first, and then stained with ethidium bromide solution $(1 \mu \mathrm{g} / \mathrm{ml})$ for $20 \mathrm{~min}$ to enable the unlabelled DNA to be visualized.

\section{Results and discussion}

\section{Preparation of azide and trans-cyclooctene modified triphosphates}

Modified deoxynucleoside triphosphates were synthesized by the Yoshikawa procedure. ${ }^{20}$ Phosphorus oxychloride $\left(\mathrm{POCl}_{3}\right)$ in trimethylphosphate was employed to generate the 5'dichlorophosphate derivative of the nucleoside, and the reaction mixtures were treated with tetrabutylammonium pyrophosphate to produce the required triphosphates. The reactions were carried out at $-15{ }^{\circ} \mathrm{C}$ to minimize the production of byproducts. 5-Azidomethyl dUTP (Scheme 1.A) and 5aminopropargyl-dUTP ${ }^{14}$ were obtained by this method. 5Aminopropargyl-dUTP was labelled with the two active esters of trans-cyclooctene (structures in SI Figure S-1) to yield two TCO-modified triphosphates with different length of linkage (Scheme 1.B).

\section{Primer extension reactions}

The efficiency of incorporation of the azidomethyl and TCO dUTPs in primer extension experiments was evaluated using three different DNA templates (Table 1). Template T1 has three adenine sites for incorporation of the modified dUTPs, and these are well separated. The more challenging template T2 carries six such sites, four of which are clustered together, presenting a particularly difficult region for the polymerase to incorporate unnatural dNTPs against. 6-Carboxyfluorescein (FAM) was attached to the 5 '-end of the primer for ease of visualization in gel electrophoresis analysis. Polyacrylamide gel electrophoresis (PAGE) and mass spectrometry confirmed that KOD and GoTaq thermostable polymerases and Klenow large fragment DNA polymerase all successfully incorporated azidomethyl dUTP (AM dUTP) into the templates T1 and T2 (Figure 2 and SI Figure S-2 and S-3, Table S-1). In some control reactions in which dTTP or modified dUTP was absent, single nucleotide extension products were observed due to nucleotide misincorporation. The resultant 3'-mismatched

complex could not extend further and two closely running bands were observed on PAGE gels (e.g. Figure 2 lane $\mathrm{N}$ and Figure 3 dNTP lane 1).

A

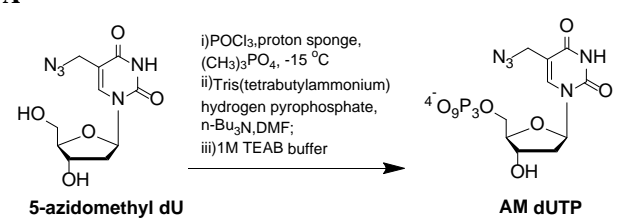

B

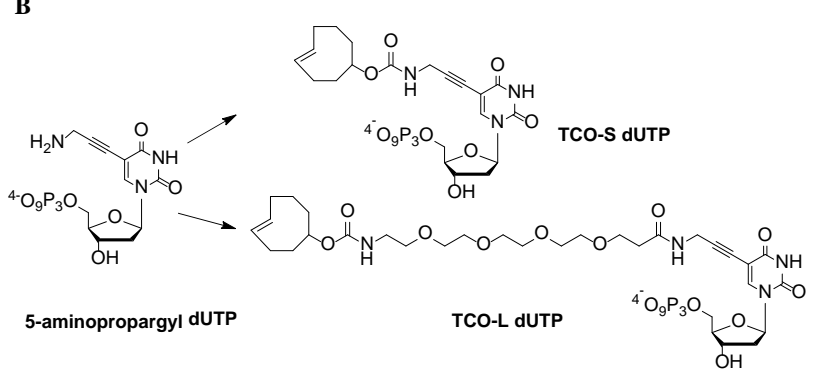

C
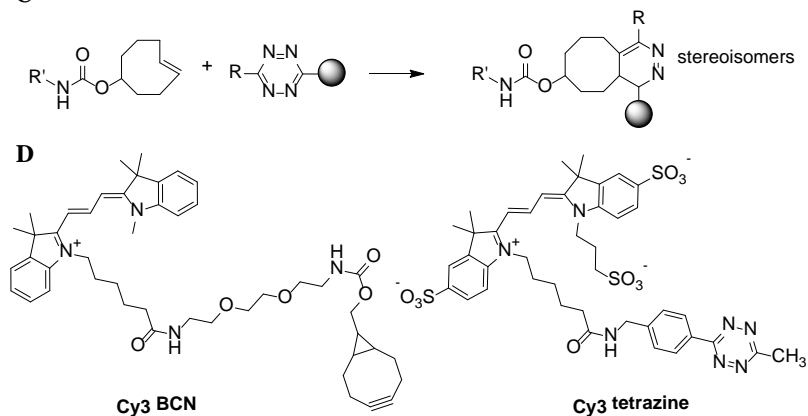

Scheme 1. Synthesis of A). AM dUTP and B). TCO-S dUTP and TCO-L dUTP; C). General reaction of cyclooctene with a tetrazinederivatised fluorescent dye; D). Cy3 derivatives used in this study.

$\mathbf{A}$

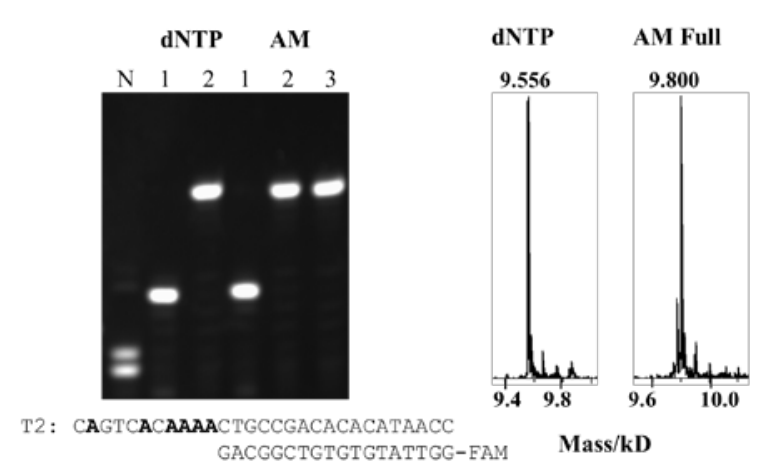

Figure 2. Successful primer extension using azidomethyl dUTP (AM dUTP) with Klenow polymerase, primer P4 and template T2. 3.2 nmol of each triphosphate was used unless otherwise stated. A). Lane N, primer and template with dATP, dCTP and dGTP but without dTTP; dNTP lanes, unmodified triphosphate reactions; AM lanes, modified AM dUTP reactions; Lane 1, dTTP or modified dUTP; Lane 2, dTTP or modified dUTP + dATP + dCTP + dGTP; Lane 3, dTTP + modified dUTP (1.6 nmol of each) + dATP + dCTP + dGTP. (20\% PAGE gel) B). Mass spectra (MS) of fully extended primers (lanes 2 from A), unmodified dNTP product (calc. 9553, found 9556); AM dUTP product (calc. 9799, found 9800). 
For the two trans-cyclooctene modified triphosphates studied, TCO-S dUTP was consistently incorporated more efficiently than TCO-L dUTP. Polyacrylamide gel electrophoresis and mass spectrometry confirmed that KOD, GoTaq and Klenow large fragment polymerases successfully incorporated TCO-S dUTP into the templates T1 and T2 (Figure 3 and SI Figure S4, Table S-1). However, for template T2 the fully extended products of TCO-L dUTP were only obtained using GoTaq polymerase (SI Figure S-4, Table S-1), so the extended ethylene glycol chain did not confer any advantages, in fact it was detrimental. As anticipated, copies of the templates containing TCO dUTPs exhibited lower electrophoretic mobility compared to the natural counterparts (Figure 3, lanes $1,2)$. This is due to the extra bulk of the modified uracil bases, particularly TCO-L dUTP.

A

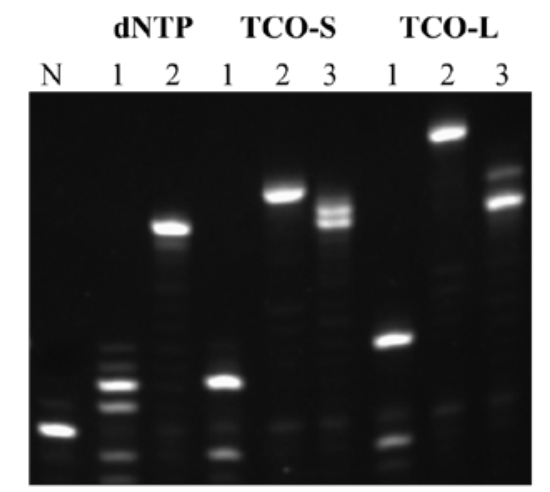

T1: CAGTCACTGTACTGCCGACACACATAACC GACGGCTGTGTGTATTGG-FAM

B

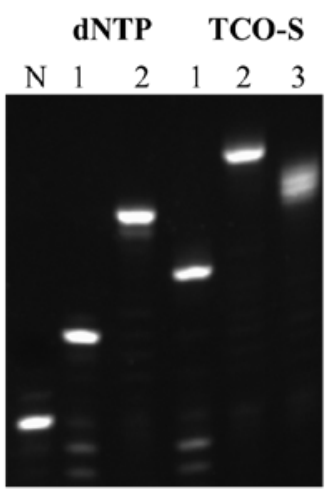

T2: CAGTCACAAAACTGCCGACACACATAACC GACGGCTGTGTGTATTGG-FAM

Figure 3. Successful primer extension using trans-cyclooctene dUTPs with Klenow DNA polymerase. $3.2 \mathrm{nmol}$ of each triphosphate was used unless otherwise stated. A). Reaction with primer $\mathrm{P} 4$ and template $\mathrm{T} 1$; B). Reaction with primer $\mathrm{P} 4$ and template T2. Lane N, primer and template without dNTPs; dNTP lanes, unmodified triphosphate reactions; TCO-S and TCO-L lanes, modified TCO dUTP reactions; Lane 1, dTTP or modified dUTP; Lane 2, dTTP or modified dUTP + dATP + dCTP + dGTP; Lane 3, dTTP + modified dUTP (1.6 nmol of each) + dATP + dCTP + dGTP. (20\% PAGE gel)
In some applications it is advantageous to control (i.e. reduce) the density of reactive groups (and ultimately fluorophores) introduced via the modified dUTP to avoid fluorescence quenching in the labelled DNA. This can be done using a mixture of the modified dUTP and dTTP in the same extension reaction. This will only be successful if the modified dUTP competes with dTTP for incorporation. To confirm this is the case for the modified dUTPs we carried out linear copying to explore the consequences of mixing them with dTTP in the presence of dATP, dCTP and dGTP (Figures 2.A and 3, lane 3, SI Figure S-5). The modified triphosphates were all able to compete with dTTP, producing fully extended primers with mixtures of dTTP and modified triphosphate opposite to the dA sites in the templates. This is evident from the multiple or broad bands on the gels and from the mass spectra of the fully extended products (SI Figure S-5).

\section{Fluorescent labelling}

The products of single nucleotide primer extension were fluorescently labelled to determine the efficiency of single terminal base labelling. The AM dUTP linear extension product was labelled with Cy3-bicyclo [6.1.0] non-4-yne (Cy3-BCN) via the strain-promoted cycloaddition (SPAAC) reaction and the trans-cyclooctene functionalized product was labelled with 6-methyl-tetrazine-sulfo-Cy3 via the inverse electron-demand Diels-Alder (iEDDA) reaction (Scheme 1.C and D). The latter reaction went to completion within $30 \mathrm{~min}$, whereas for AM dUTP, some unlabelled starting material remained after $1 \mathrm{~h}$ (Figure 4). Primer extension reaction products obtained from dTTP were employed as negative controls for the labelling reactions and gel mobility studies. The labelled products, which were characterized by mass spectrometry (Figure 5), exhibited much lower electrophoretic mobility than the unlabeled controls (Figure 4).

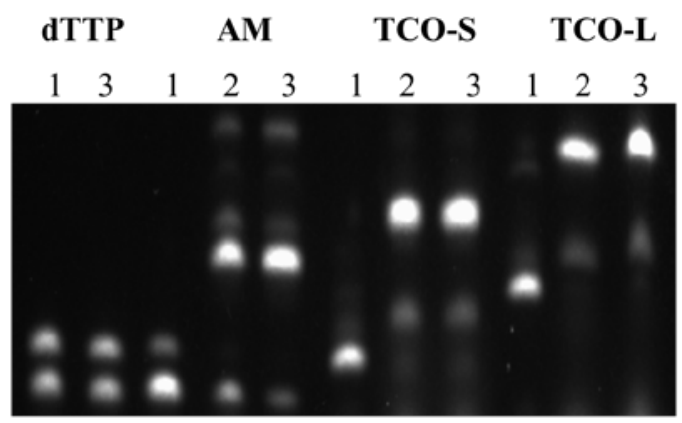

T1: CAGTCACTGTACTGCCGACACACATAACC TGACGGCTGTGTGTATTGG-EAM

Figure 4. Efficient fluorescent labelling of single nucleotide primer extension products obtained from modified dUTPs only, GoTaq polymerase, primer P4 and template T1. dTTP and AM dUTP products were labelled with Cy3-BCN; TCO dUTPs products were labelled with 6-methyl-tetrazine-sulfo-Cy3. The bold 3'-T in the extended primer contains either thymidine or the modified nucleotide. Lane 1, unlabelled products; Lane 2, 30 min labelling; Lane 3, 60 min labelling. (20 \% PAGE gel)

When the labelling reactions were carried out on fully extended products (multiple labelling), the products containing the AM dU nucleotide exhibited much lower labelling efficiency (SI Figure S-6). This is in accordance with the known rate constants for the two reactions. The trans-cyclooctene-tetrazine 
is the fastest known bioorthogonal click reaction with a secondorder rate constant up to $22000 \mathrm{M}^{-1} \mathrm{~s}^{-1}$. This is several orders of magnitude faster than the SPAAC reaction (which in turn is much faster than the CuAAC reaction). ${ }^{21}$ It is important to bear in mind that these reported kinetic data were obtained from small molecule studies; in the context of DNA labelling various factors such as steric hindrance from the oligonucleotide chain, and clustering of labelled dU residues (sequence context) influence reaction rates. Moreover, when selecting click reactions on the basis of reaction rates it should be stated that the CuAAC reaction can be accelerated by addition of $\mathrm{Cu}(\mathrm{I})$ binding ligands ${ }^{22}$ and the SPAAC reaction rate can be greatly increased by the use of electron deficient aryl azides. ${ }^{23}$ It should also be noted that some "click" reactions are not truly bioorthogonal, for example cyclooctynes can react with nucleophiles such as thiols which are prevalent in cells, thereby sequestering reactants. ${ }^{5}$
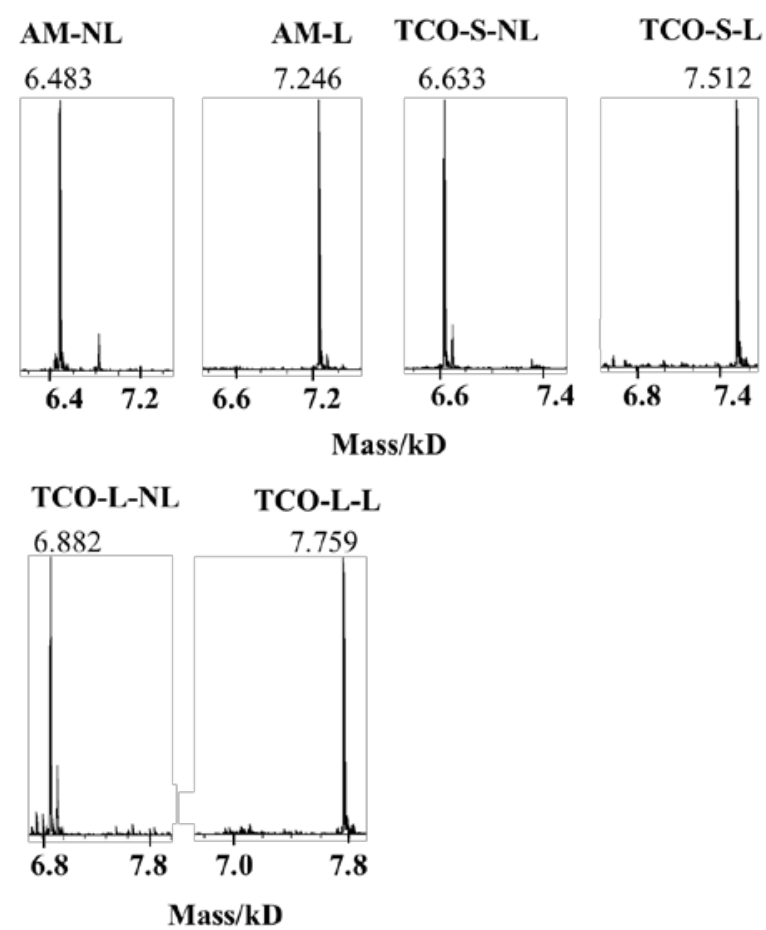

Figure 5. Mass Spectrometry characterization of single fluorescently labelled nucleotide primer extension products obtained from modified dUTPs only, GoTaq polymerase, primer P4 and template T1. AM dUTP products were labelled with Cy3-BCN; TCO dUTPs products were labelled with 6-methyl-tetrazine-sulfo-Cy3. AM-NL (not labelled): calc. 6483, found 6483; AM-L (labelled with Cy3BCN): calc. 7246, found 7246; TCO-S-NL: calc. 6633, found 6633; TCO-S-L: calc. 7513, found 7512; TCO-L-NL: calc. 6880, found 6882; TCO-L-L: calc. 7760, found 7759. (NL = not labelled, $\mathrm{L}=$ labelled).

\section{Reverse transcription}

It is important for biological imaging and diagnostic studies to develop methods to produce fluorescently labelled cDNA probes from RNA molecules of biological origin. To this end our three modified dUTPs were tested for incorporation against

a synthetic RNA template employing Moloney Murine Leukemia Virus reverse transcriptase (M-MuLV RT, RNase $\mathrm{H}^{-}$) and Avian Myeloblastosis Virus reverse transcriptase (AMV RT). The two RNA templates T5 and T6 have the equivalent sequence to the previously used DNA template. AM dUTP and TCO-S dUTP were incorporated efficiently into both templates to give fully extended products with both reverse transcriptase enzymes (Figure 6 and SI Figure S-7 to S-9, Table S-1). All modified dUTPs were incorporated more efficiently with MMuLV RT (RNase $\mathrm{H}^{-}$) than with AMV RT (SI Figure S-7, S-9).

\section{Polymerase chain reaction}

In the above linear copying studies we have demonstrated that the AM and TCO dUTPs can be incorporated into DNA by primer extension. The generation of PCR amplification products that contain dNTP analogues is another important method to prepare fluorescent probes. This requires the chemical modifications to be tolerated by DNA polymerases when they are in the template strand as well as when they are attached to dUTP for incorporation into the template. We have investigated this with our AM dUTP and TCO dUTP analogues. Initial PCR reactions were carried out using 81-mer DNA template T9. In all cases amplification was successful, even when using the modified triphosphate in combination with dATP, dCTP and dGTP with no added dTTP (Figures 7.A, lane TA4 and Figure 8.A, lane TS4, TL4). Melting experiments on the amplicons showed that the AM and TCO-L modifications destabilize the DNA duplex in a manner proportional to the number of modifications in the PCR products, whereas TCO-S does not (Figure 7.B and 8.B). Such effects of modified nucleotides on duplex stability should be evaluated and taken into account when designing PCR protocols and cycles for incorporation of modified dNTPs.

$\mathbf{A}$

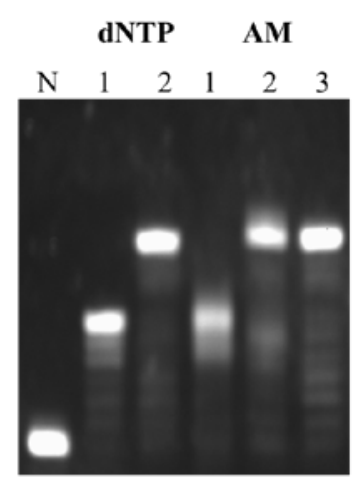

T6: CAGUCACAAAACUGCCGACACACAUAACC GACGGCTGTGTGTATTGG-FAM

Figure 6. Efficient reverse transcription reactions using M-MuLV (RNase $\mathrm{H}^{-}$) reverse transcriptase with primer $\mathrm{P} 4$ and template T6. 3.2 nmol of each triphosphate were used unless otherwise stated. A). Reactions with AM dUTP; B). Reactions with TCO-S dUTP. Lane $\mathrm{N}$, primer and template without dNTPs; dNTP lanes, unmodified triphosphate reactions; AM lanes, modified AM dUTP reactions; TCO-S lanes, modified TCO-S dUTP reactions; Lane 1, dTTP or modified dUTP; Lane 2, dTTP or modified dUTP + dATP + dCTP + dGTP; Lane 3, dTTP + modified dUTP (1.6 nmol of each) + dATP + dCTP + dGTP. (20\% PAGE gel) 
A

\section{B}

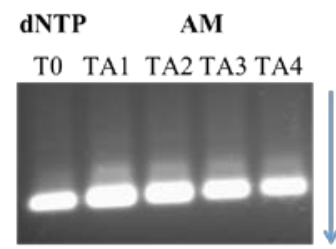

AM dUTP(\%) $\quad 25 \% \quad 50 \% 75 \% 100 \%$

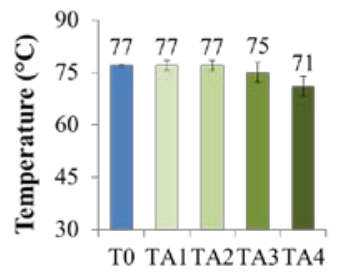

Figure 7. A). Efficient PCR amplification with different percentages of AM dUTP relative to dTTP $(5 \mathrm{nmol}$ in total of AM dUTP/dTTP + $5 \mathrm{nmol}$ each of dATP $+\mathrm{dCTP}+\mathrm{dGTP}$ ) using GoTaq polymerase and template T9. Lane T0, unmodified amplicon; lane TA1 to TA4, $25 \%$ to $100 \%$ modified AM dUTP relative to dTTP. (2 \% agarose gel) B). Melting temperatures of PCR amplicons.

Encouraged by these results, PCR amplification of a more demanding 523 bp region of a plasmid with a total of 335 thymidine sites was evaluated. Amplification was attempted with AM dUTP along with dATP, dCTP and dGTP in the absence of dTTP (Figure 9). Although initial PCR with this template using $1 \mathrm{mM} \mathrm{MgCl}_{2}$ was inefficient the problem was easily solved by increasing the $\mathrm{Mg}^{2+}$ ion concentration to achieve efficient amplification. This requirement for higher $\mathrm{Mg}^{2+}$ concentration might be due to the positive effect of $\mathrm{Mg}^{2+}$ on the stability of the duplex formed between the extended primer and the template.

A

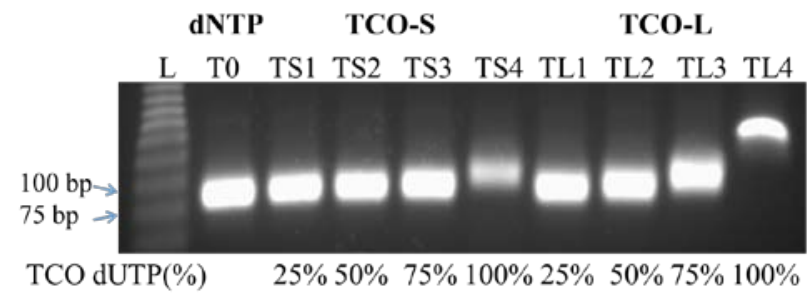

B

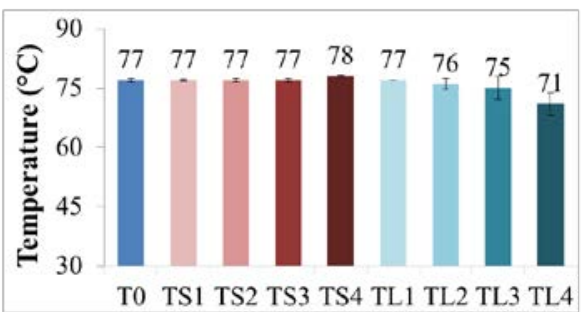

Figure 8. A). Efficient PCR amplification with different percentages of TCO dUTPs relative to dTTP (5 nmol in total of TCO dUTP/dTTP + 5 nmol each of dATP + dCTP + dGTP) using GoTaq polymerase and template T9. Lane L, 25 bp ladder; lane T0, unmodified amplicon; lane TS1/TL1 to TS4/TL4, $25 \%$ to $100 \%$ modified TCO-S/TCO-L dUTP relative to dTTP. (2 \% agarose gel) B). Melting temperatures of PCR amplicons.

The efficiency of PCR amplification of plasmid template T12 was sensitive to TCO-S dUTP and more so to TCO-L dUTP. We varied the percentage of dTTP and were able to obtain reasonable yields of PCR amplicons with $50 \%$ TCO-S dUTP and $25 \%$ TCO-L dUTP (Figure 10.A, lane PS2 and PL1). As observed with the previous shorter amplicon, DNA duplex stability was affected less by TCO-S dUTP than by TCO-L dUTP. Although it might be possible to optimize the PCR reaction for incorporation of higher percentages of TCO-L, the use of TCO-S is a better option for introduction of transcyclooctene into DNA.
A

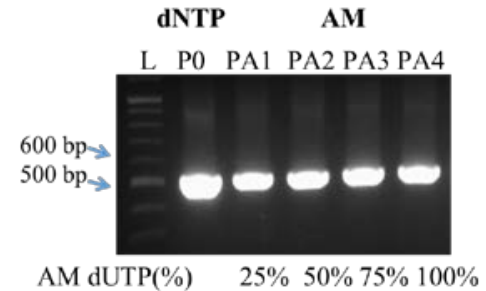

B

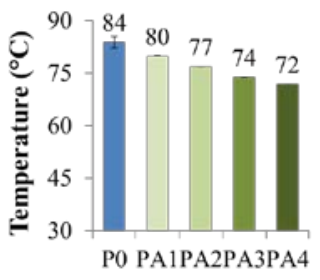

Figure 9. A). Efficient PCR amplification with different percentages of AM dUTP relative to dTTP (10 nmol in total of AM dUTP/dTTP $+10 \mathrm{nmol}$ each of dATP + dCTP + dGTP) using KOD polymerase and template T12 (plasmid template). Lane L, 100 bp ladder; lane P0, unmodified amplicon; lane PA1 to PA4, $25 \%$ to $100 \%$ modified AM dUTP relative to dTTP. (1.5\% agarose gel) B). Melting temperatures of PCR amplicons.

Labelling the PCR products containing trans-cyclooctene dUTPs with 6-methyl-tetrazine-sulfo-Cy3 gave the expected pink bands as shown in Figure 11.B for the 523 bp labelled product. The labelled products migrated slower in a manner proportionate to the percentage of modified dUTP used in the PCR amplification (Figure 11.A and SI Figure S-14).
A

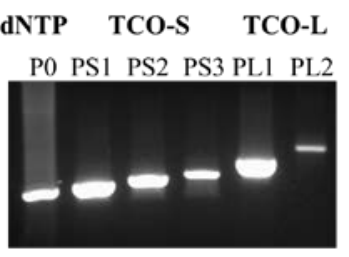

TCO dUTP(\%) $25 \% 50 \% 75 \% 25 \% 50 \%$
B

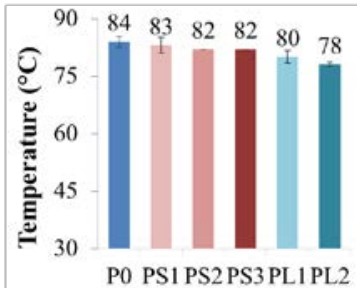

Figure 10. A). Efficient PCR amplification with different percentages of TCO dUTP relative to dTTP $(10 \mathrm{nmol}$ in total of TCO dUTP/dTTP + $10 \mathrm{nmol}$ each of dATP + dCTP + dGTP) using KOD polymerase and template T12 (plasmid template). Lane P0, unmodified amplicon; lane PS1 to PS3, $25 \%$ to $75 \%$ modified TCO-S dUTP relative to dTTP; lane PL1 to PL2 $25 \%$ to $50 \%$ modified TCO-L dUTP relative to dTTP. (1.5\% agarose gel) B). Melting temperatures of PCR amplicons.

\section{Conclusions}

5-Azidomethyl dUTP and two trans-cyclooctene dUTPs have been successfully incorporated into DNA by primer extension, reverse-transcription and PCR amplification. PCR was successful with AM dUTP even when the modified dUTP was not supplemented with dTTP. In general AM dUTP is a better substrate than the TCO dUTPs for the DNA polymerases used in this study, and TCO-S dUTP with a short linker is incorporated by DNA polymerases more efficiently than the 
longer version. The iEDDA click reaction between cyclooctene and tetrazine is more efficient than the SPAAC reaction between azide and BCN, as would be expected from its much faster reported kinetics. ${ }^{21,24,25}$

A

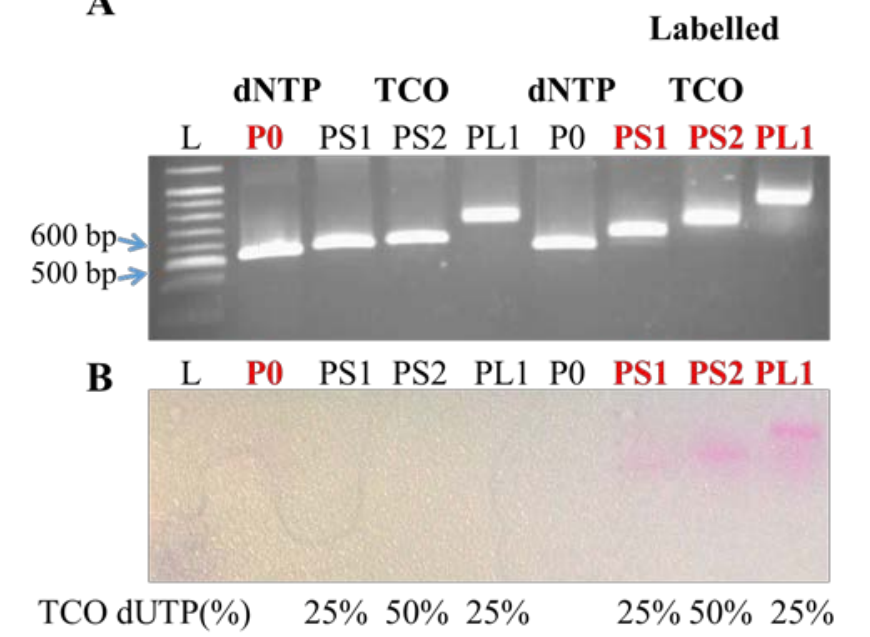

Figure 11. Fluorescent labelling of PCR products obtained from different percentages of dTTP and TCO dUTPs $(10 \mathrm{nmol}$ in total of TCO dUTP/dTTP + $10 \mathrm{nmol}$ each of dATP + dCTP + dGTP) using KOD polymerase and template T12 (plasmid template). Lane L, 100 bp ladder; lane P0, unmodified amplicon; lane PS1 to PS2, $25 \%$ to 50 \% modified TCO-S dUTP relative to dTTP; lane PL1, $25 \%$ modified TCO-L dUTP relative to dTTP. Labels above gels in black are for products without 6-methyl-tetrazine-sulfo-Cy3, while labels in bold red are for products that have been reacted with 6-methyltetrazine-sulfo-Cy3. The $\mathrm{P} 0$ red negative control is positioned distant from the Cy3-labelled products to illustrate that contamination with free dye is not a significant problem. A). Fluorescent gel was visualized by staining with ethidium bromide; B). Gel picture was taken under normal light. (1.5 \% agarose gel)

Very recently we have investigated the use of 5-azidomethyl $\mathrm{dU}$ (AM dU) and TCO dU to label nascent genomic DNA in cell culture with fluorescent cyclooctynes, and compared this directly with ethynyl dU incorporation followed by labelling with fluorescent azides. The ethynyl dU experiments yielded excellent fluorescent images whereas the AM dU and TCO dU studies gave disappointing results, with almost no detectable fluorescence. There are several possible reasons for this and further detailed studies are in progress.

\section{Acknowledgements}

TB and AHE-S are grateful to the UK BBSRC for funding via the sLOLA grant BB/J001694/1: "Extending the Boundaries of Nucleic Acid Chemistry."

\section{Notes and references}

${ }^{a}$ Department of Chemistry, University of Oxford, Chemistry Research Laboratory, 12 Mansfield Road, Oxford, OX1 3TA, UK

${ }^{b}$ Chemistry Branch, Department of Science and Mathematics, Faculty of Petroleum and Mining Engineering, Suez University, Suez, 43721, Egypt

$\dagger$ Electronic Supplementary Information (ESI) available: Synthesis of modified triphosphates and Cy3-BCN. MS and NMR spectra characterization. PAGE and mass spectra results of primer extension and reverse transcription. Melting experiments and labelling experiments of PCR products. See DOI: 10.1039/b000000x/

1.

2.

3.

4.

5.

6.

7.

8.

8.

9.

10.

11.

12.
C. W. Tornoe, C. Christensen and M. Meldal, J. Org. Chem., 2002, 67, 3057-3064.

V. V. Rostovtsev, L. G. Green, V. V. Fokin and K. B. Sharpless, Angew. Chem. Int. Edit., 2002, 41, 2596-2599.

A. Salic and T. J. Mitchison, Proc. Nat. Acad. Sci. U.S.A. , 2008, $105,2415-2420$

C. Y. Jao and A. Salic, Proc. Nat. Acad. Sci. U.S.A. , 2008, 105, 15779-15784.

M. F. Debets, S. S. Van Berkel, J. Dommerholt, A. J. Dirks, F. Rutjes and F. L. Van Delft, Accounts Chem. Res., 2011, 44, 805815.

J. Dommerholt, S. Schmidt, R. Temming, L. J. A. Hendriks, F. Rutjes, J. C. M. van Hest, D. J. Lefeber, P. Friedl and F. L. van Delft, Angew. Chem. Int. Edit., 2010, 49, 9422-9425.

A. B. Neef and N. W. Luedtke, Chembiochem, 2014, 15, 789-793.

J. C. Jewett and C. R. Bertozzi, Chem. Soc. Rev. , 2010, 39, 12721279.

M. Shelbourne, T. Brown, Jr., A. H. El-Sagheer and T. Brown, Chem. Commun., 2012, 48, 11184-11186.

A. H. El-Sagheer and T. Brown, Chem. Soc. Rev. , 2010, 39, 1388-1405.

J. Gierlich, K. Gutsmiedl, P. M. E. Gramlich, A. Schmidt, G. A. Burley and T. Carell, Chem. Eur. J., 2007, 13, 9486-9494.

F. Seela, V. R. Sirivolu and P. Chittepu, Bioconj. Chem., 2008, 19, 211-224.

S. Obeid, H. Busskamp, W. Welte, K. Diederichs and A. Marx, Chem. Commun., 2012, 48, 8320-8322.

X. Ren, M. Gerowska, A. H. El-Sagheer and T. Brown, Bioorg. Med. Chem., 2014, 22, 4384-4390.

H. Rao, A. A. Tanpure, A. A. Sawant and S. G. Srivatsan, Nat. Protoc. , 2012, 7, 1097-1112.

H. Rao, A. A. Sawant, A. A. Tanpure and S. G. Srivatsan, Chem. Commun. , 2012, 48, 498-500.

M. L. Winz, A. Samanta, D. Benzinger and A. Jaschke, Nucleic Acids Res. , 2012, 40, 13.

S. H. Weisbrod and A. Marx, Chem. Commun. , 2007, 18, 18281830.

S. Obeid, A. Baccaro, W. Welte, K. Diederichs and A. Marx, Proc. Natl. Acad. Sci. U. S. A., 2010, 107, 21327-21331.

M. Yoshikawa, T. Kato and T. Takenishi, Tetrahedron Lett., 1967, 50, 5065-5068.

A. C. Knall and C. Slugovc, Chem. Soc. Rev. , 2013, 42, 51315142.

T. R. Chan, R. Hilgraf, K. B. Sharpless and V. V. Fokin, Org. Lett., 2004, 6, 2853-2855.

J. Dommerholt, O. van Rooijen, A. Borrmann, C. F. Guerra, F. M. Bickelhaupt and F. L. van Delft, Nat. Commun., 2014, 5, 5378.

D. Wang, W. Chen, Y. Zheng, C. Dai, K. Wang, B. Ke and B. Wang, Org. Biomol. Chem., 2014, 12, 3950-3955.

K. Wang, D. Wang, K. Ji, W. Chen, Y. Zheng, C. Dai and B. Wang, Org. Biomol. Chem., 2015, 3, 909-915. 


\section{TOC graphic}

Azide and trans-Cyclooctene dUTPs: Incorporation into DNA Probes and Fluorescent Click-Labelling

Xiaomei Ren, Afaf H. El-Sagheer and Tom Brown

Azidomethyl and cyclooctene dUTPs have been incorporated into DNA by various polymerase enzymes and labelled with cyclooctyne and tetrazine fluorophores
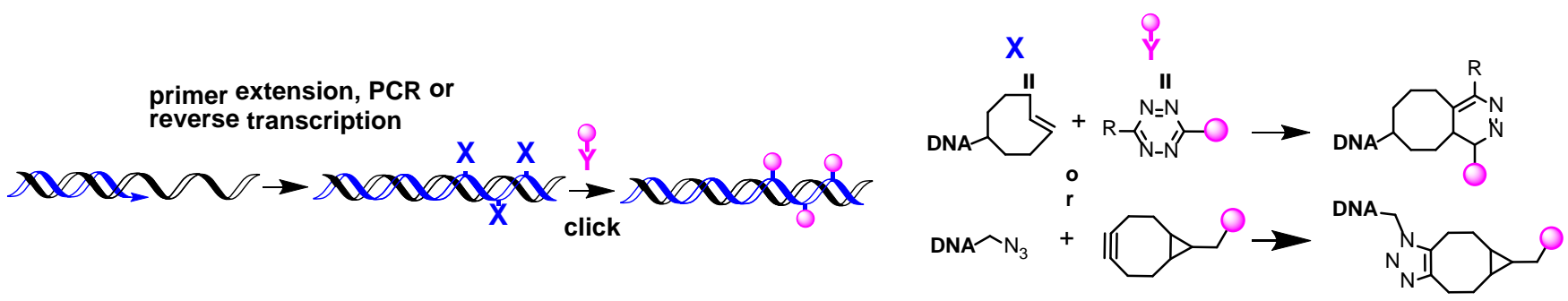